\title{
Cold-Pressed Jute Fibre Composite Tensile Properties Effect on Hygrothermal Treatment
}

\author{
Nagaraj ${ }^{1}$, Khasim Sharif $\mathrm{Sk}^{2,}$ D.Jagadish ${ }^{3}$, K. Phaneendra ${ }^{4}$ \\ ${ }_{1,2}^{\text {(Assistant professor, Mechanical Department, CMRTC/ JNTUH, India) }}$ \\ ${ }^{3}$ (Professor, Mechanical Department, VFSTR, India) \\ ${ }^{4}$ (Professor\& principal, Mechanical Department, VLITS, India)
}

\begin{abstract}
The growing environment awareness demands the use of nature fibres as reinforcement materials in commercial. The natural fibres however are hydrophilic in nature and their composites undergo environmental degradation during service, hydrothermal conditioning on as received jute fabric at different temperatures of cold press has been studied in the present investigation. These composites are usually subjected to various loading conditions. Therefore, an attempt has been made to study the tensile properties of the composites. Fractography studies were carried out to study the fracture surface. It is noticed that the major mode of failure is due to fiber pullout and matrix cracking. The result from the hydrothermal studies shows the decrease in strength values of the composites on prolong exposure to humid
\end{abstract}

Keywords: Cashew nut shell, Cold pressed jute, hydrothermal treatment, Silica sand

\section{Introduction}

Over the last thirty years composite materials, plastics and ceramics have been the dominant emerging materials. The volume and number of applications of composite materials have grown steadily, penetrating and conquering new markets relentlessly [1]. Modern composite materials constitute a significant proportion of the engineered materials market ranging from everyday products to sophisticated niche applications. The composites industry has begun to recognize that the commercial applications of composites promise to offer much larger business opportunities than the aerospace sector due to the sheer size of transportation industry. Thus the shift of composite applications from aircraft to other commercial uses has become prominent in recent years. Unlike conventional materials (e.g., steel), the properties of the composite material can be designed considering the structural aspects [2]. The design of a structural component using composites involves both material and structural design. Composite properties (e.g. stiffness, thermal expansion etc.) can be varied continuously over a broad range of values under the control of the designer. Kelly very clearly stresses that the composites should not be regarded simple as a combination of two materials. In the broader significance; the combination has its own distinctive properties [3]. In terms of strength to resistance to heat or some other desirable quality, it is better than either of the components alone or radically different from either of them.

Beghezan defines as "The composites are compound materials which differ from alloys by the fact that the individual components retain their characteristics but are so incorporated into the composite as to take advantage only of their attributes and not of their short comings", in order to obtain improved materials[4]. It explains composite materials as heterogeneous materials consisting of two or more solid phases, which are in intimate contact with each other on a microscopic scale. They can be also considered as homogeneous materials on a microscopic scale in the sense that any portion of it will have the same physical property

\section{Components of A Composite Material}

In its most basic form a composite material is one, which is composed of at least two elements working together to produce material properties that are different to the properties of those elements on their own. In practice, most composites consist of a bulk material (the "matrix"), and a reinforcement of some kind, added primarily to increase the strength and stiffness of the matrix.

\subsection{Role of matrix in a composite}

Many materials when they are in a fibrous form exhibit very good strength property but to achieve these properties the fibers should be bonded by a suitable matrix. The matrix isolates the fibers from one another in order to prevent abrasion and formation of new surface flaws and acts as a bridge to hold the fibers in place. A good matrix should possess ability to deform easily under applied load, transfer the load onto the fibers and evenly distributive stress concentration. 


\subsection{Materials used as matrices in composites}

In its most basic form a composite material is one, which is composed of at least two elements working together to produce material properties that are different to the properties of those elements on their own. In practice, most composites consist of a bulk material (the matrix) and a reinforcement of some kind, added primarily to increase the strength and stiffness of the matrix.
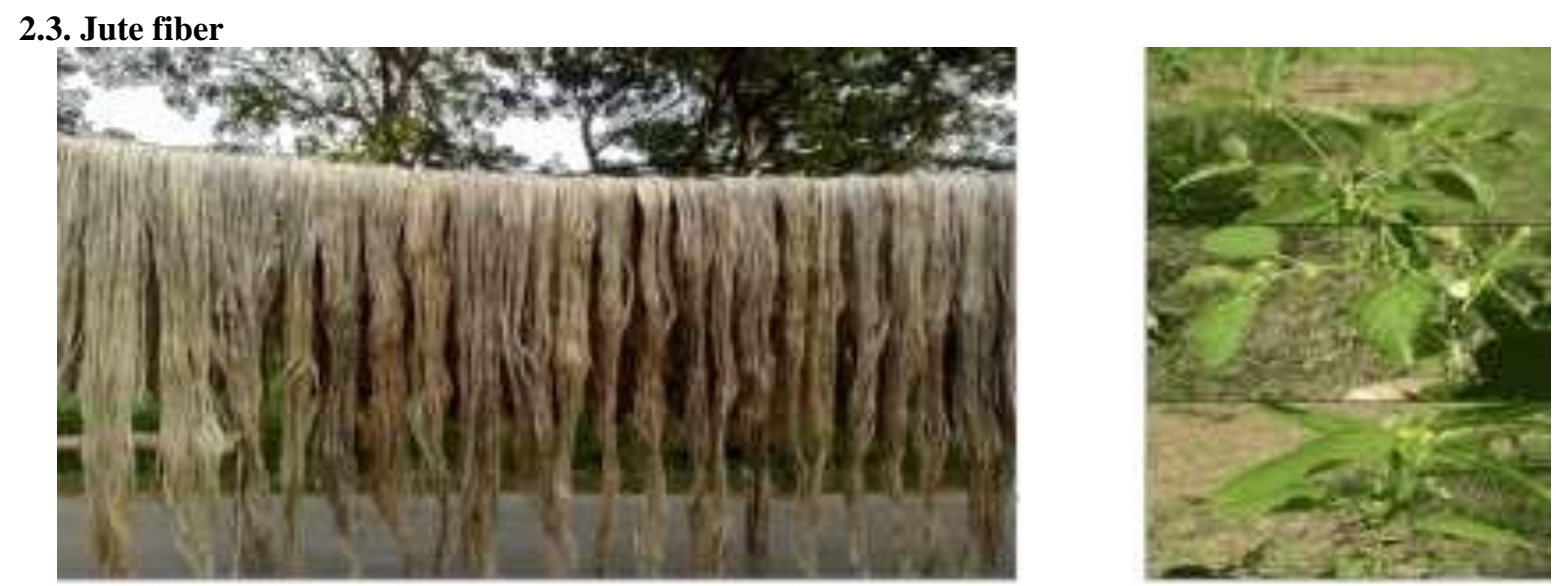

Figure-1; Jute Fiber

Lignocelluloses fibers are favorably bonded with phenolic resin to have better water resistance rather than urea or melamine resin. Hence, water soluble phenol formaldehyde resin was selected for the development of rigid jute board for good serviceable mechanical properties. To achieve better wet ability of jute with resin and to improve strength properties, fiber pre-treatment is necessary. Simple pre-treatment is done with lowcondensed resins like melamine resin, phenolic resin and CNSL modified phenol formaldehyde resin. Indicative physical properties composites from untreated \& pre-treated jute nonwoven with PF resin are shown in Table Jute as other lignocelluloses fibers consists of $-\mathrm{OH}$ group which causes it susceptible to moisture and directly impairs the properties of jute composite specially dimensional stability. Due to this polar group, jute also is not efficiently adhered to non-polar matrices. To overcome this difficulties this fiber should be modified chemically or hydro-thermally. To improve the interface adhesion between the non polar matrices and hydrophilic fiber, coupling agent or compatibiliser should be used.
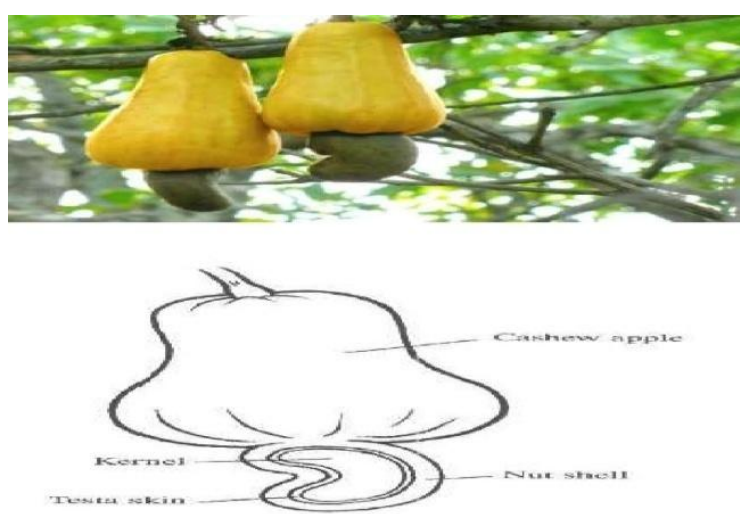

Figure-2: Cashew Nut Shell

III. Cashew Nut Shell

Cashew (Anacardium occidentale) is an important plantation crop of India. India has the largest area under cashew (9.23 lakh ha) and stands as the second largest producer of cashew (7.00 lakh MT) in the world (CEPC, 2012). Today, India is the 2nd largest processor and exporter of cashew in the world..Maharashtra ranks first in the production ( $28.78 \%$ of the country) and productivity of cashew nut in India. The cashew nut consists of kernel, shell and testa. It contains on an average 20 to $22 \%$ kernel (edible portion), 2-5 \% testa and $65-75 \%$ shell (outer covering). The cashew nut shell contains $25-30 \%$ dark reddish brown viscous phenolic liquid known as Cashew Nut Shell Liquid and abbreviated as CNSL. 


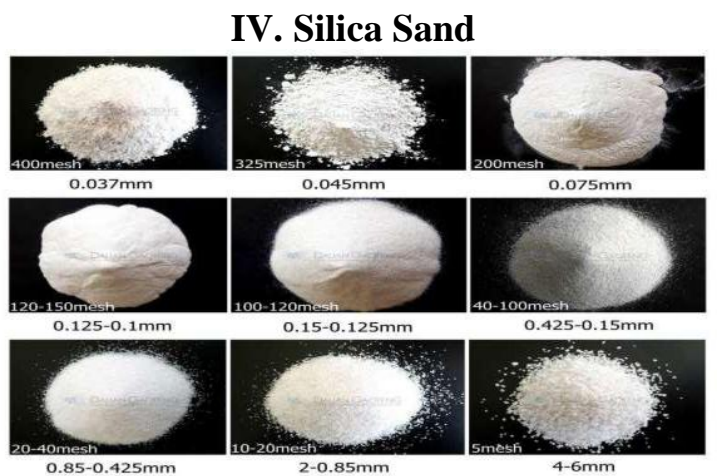

Figure-3: silica sand

Sand is a naturally occurring granular material composed of finely divided rock and mineral particles. The composition of sand is highly variable, depending on the local rock sources and conditions, but the most common constituent of sand in inland continental settings and non- tropical coastal settings is silica (silicon dioxide, or $\mathrm{SiO} 2$ ), usually in the form of quartz. The second most common type of sand is calcium carbonate, for example aragonite, which has mostly been created, over the past half billion years, by various forms of life, like coral and shellfish. In terms of particle size as used by geologists, sand particles range in diameter from $0.0625 \mathrm{~mm}$ (or $1 / 16 \mathrm{~mm}$ ) to $2 \mathrm{~mm}$. An individual particle in this range size is termed a sand grain. Sand grains are between gravel (with particles ranging from $2 \mathrm{~mm}$ up to $64 \mathrm{~mm}$ ) and silt (particles smaller than $0.0625 \mathrm{~mm}$ down to $0.004 \mathrm{~mm})$.

ISO 14688 grades sands as fine, medium and coarse with ranges $0.063 \mathrm{~mm}$ to $0.2 \mathrm{~mm}$ to $0.63 \mathrm{~mm}$ to $2.0 \mathrm{~mm}$. In the United States, sand is commonly divided into five sub-categories based on size: very fine sand $(1 / 16-1 / 8 \mathrm{~mm}$ diameter), fine sand $(1 / 8 \mathrm{~mm}-1 / 4 \mathrm{~mm})$, medium sand $(1 / 4 \mathrm{~mm}-1 / 2 \mathrm{~mm})$, coarse sand $(1 / 2 \mathrm{~mm}$ $-1 \mathrm{~mm})$, and very coarse sand $(1 \mathrm{~mm}-2 \mathrm{~mm})$.

\subsection{Pictorial Procedure of preparation of cashew shell husk powder \\ V. Preparation of Specimen}

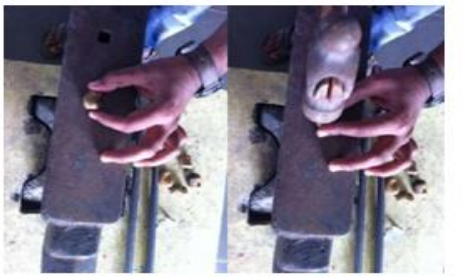

Firure-4

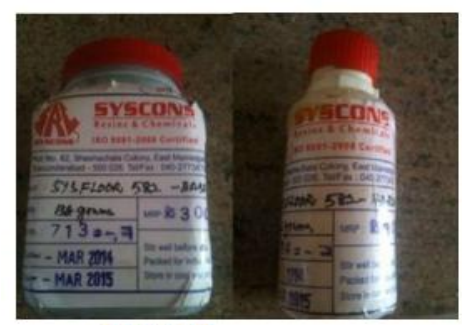

Firure-7

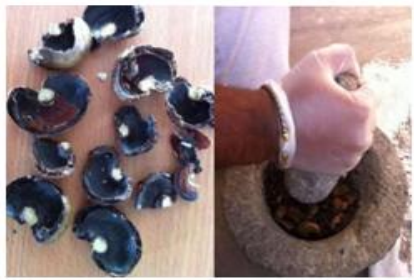

Figure-5

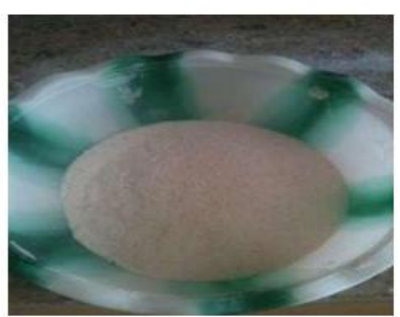

Firure-8

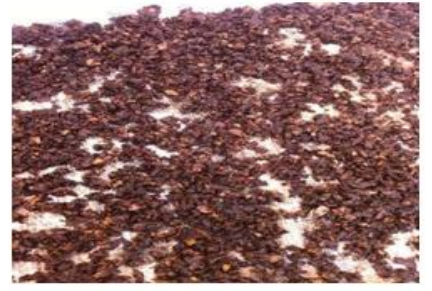

Figure-6

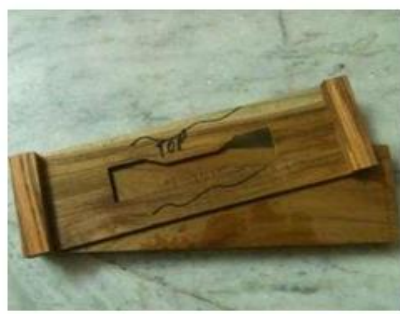

Firure-9

\subsection{Preparation procedure}

First of all, wooden moulds were prepared with the dimensions mentioned in the tensile, compression, flexural tests The mould's chamber where the composite has to be made must be coated with a thick layer which protects the resin entering into the mould. Since it is a wooden mould, its porosity can enable the wooden mould absorbing a small quantity of binder. The primer consists of base and hardener taken in the ratio 333:167. These fluids are thoroughly stirred for 5 minutes and the applied on the mould's chamber with a brush. The coating should be thick and even all over the chamber. Now, these moulds coated with primer should be dried for 8 hours to notice the formation of a thin protecting layer. The next step is a significant one by which a composite is obtained. To initiate the procedure, two major components are required.

1) Binder- Used to bind and harden the combination of various materials. 
2) filler- To strengthen the combination

In our experiment, an epoxy resin is used as a binder and silica sand, Cashew nut shell husk serves as the filler. A mandatory proportion of 138 grams of base, 62 grams hardener and $1.4 \mathrm{~kg}$ of filler has to be used as per mentioned in the SYSCONS manual for epoxy resin. The figures can be multiplied or divided according to the requirement.

The base and hardener are taken in a bowl according to the proportions mentioned above. A sensitive weighing balance is suggested for the measurement. This mixture in the bowl is thoroughly mixed. The filler is added to this mixture and mixing is done up to 5 minutes. Finally, this mixture is poured in the moulds. It should be done carefully to avoid more porosity. The upper surface is leveled.

$1^{\text {st }}$ trial: The $1^{\text {st }}$ trial was made using only the silica sand as the filler. This stood as a better trial to start the actual subject.

$2^{\text {nd }}$ trial: The second trial was made using $\mathbf{7 0 \%}$ of cashew nut shell husk and $30 \%$ of silica sand. It was failed due to the absorption of resin by the cashew nut shell husk.

$3^{\text {rd }}$ trial: In the third trial, the proportions of the base and hardener were doubled for binding the same quantity of filler. The $70 \%$ of cashew nut shell husk and $\mathbf{3 0 \%}$ of silica sand combination seemed to yield low properties.

$4^{\text {th }}$ trial: An improvisation was made by taking $\mathbf{5 0 \%}$ of cashew nut shell husk and $50 \%$ of silica with the doubled proportion of base and hardener. This trial seemed to be supreme. This was sent to the laboratory for testing.

\subsection{Tensile specimen}

\section{Experimentation}

Consider the typical tensile specimen shown in Fig.10 It has enlarged ends or shoulders for gripping. The important part of the specimen is the gage section. The cross-sectional area of the gage section is reduced relative to that of the remainder of the specimen so that deformation and failure will be localized in this region. The gage length is the region over which measurements are made and is centered within the reduced section. The distances between the ends of the gage section and the shoulders should be great enough so that the larger ends do not constrain deformation within the gage section, and the gage length should be great relative to its diameter. Otherwise, the stress state will be more complex than simple tension. There are various ways of gripping the specimen. The end may be screwed into a threaded grip, or it may be pinned; butt ends may be used, or the grip section may be held between wedges. There are still other methods. The most important concern in the selection of a gripping method is to ensure that the specimen can be held at the maximum load without slippage or failure in the grip section. Bending should be minimized the diameter.

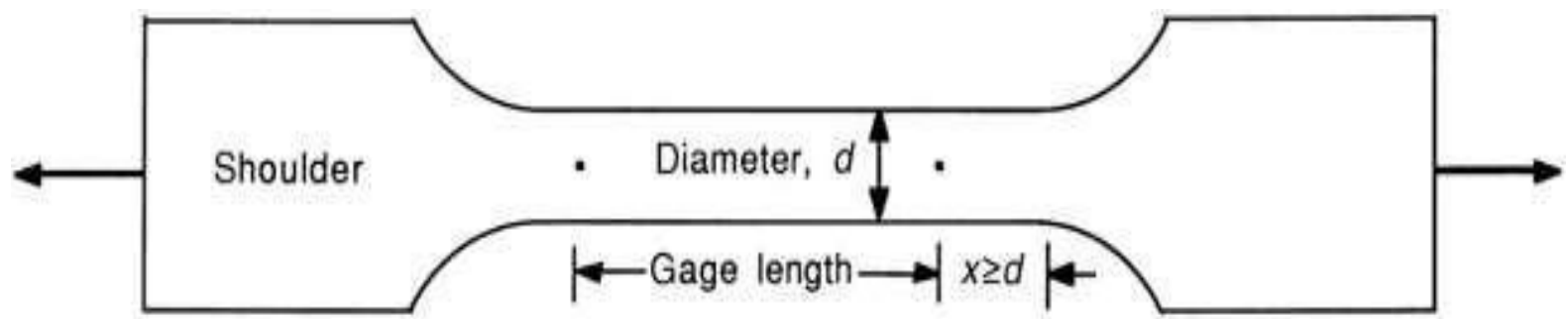

Firure-10

To avoid end effects from the shoulders, the length of the transition region should be at least as great as the diameter, and the total length of the reduced section should be at least four times

\subsection{Formulae}

Young's modulus = slope of stress Vs Strain.

Ultimate compressive strength $\quad=$ Force $(\mathrm{N})$ just before rupture / (original c/s area) Percentage reduction in length $\quad=$ (initial length- final length)*100/initial length.

\subsection{Experimentation results summary}

Table -1

\begin{tabular}{|l|l|l|l|}
\hline TEST & Sample 1 & Sample 2 & Sample 3 \\
\hline Tensile & $14.37 \mathrm{Mpa}$ & $13.16 \mathrm{Mpa}$ & $12.23 \mathrm{Mpa}$ \\
\hline Compression & $60.35 \mathrm{Mpa}$ & $61.23 \mathrm{Mpa}$ & $59.47 \mathrm{Mpa}$ \\
\hline Flexural & $22.91 \mathrm{Mpa}$ & $21.87 \mathrm{Mpa}$ & $23.51 \mathrm{Mpa}$ \\
\hline
\end{tabular}




\subsection{Deformation loads}

Table -2

\begin{tabular}{|l|l|l|l|}
\hline TEST & Sample 1 & Sample 2 & Sample 3 \\
\hline Tensile & $1484.783 \mathrm{~N}$ & $1769.42 \mathrm{~N}$ & $1404.75 \mathrm{~N}$ \\
\hline Compression & $38935.406 \mathrm{~N}$ & $39503.146 \mathrm{~N}$ & $38367.665 \mathrm{~N}$ \\
\hline Flexural & $97.71 \mathrm{~N}$ & $90.55 \mathrm{~N}$ & $119.24 \mathrm{~N}$ \\
\hline
\end{tabular}

\subsection{Wear rate results}

Table -3

\begin{tabular}{|l|l|l|}
\hline Parameter & Cashew nut shell husk & Silica sand composite \\
\hline & composite & \\
\hline Length of specimen worn out & $0.086 \mathrm{~cm} / \mathrm{min}$ & $0.12 \mathrm{~cm} / \mathrm{min}$ \\
\hline Worn out volume & $0.554 \mathrm{~cm}^{3} / \mathrm{min}$ & $0.774 \mathrm{~cm} / \mathrm{min}$ \\
\hline Density of the composite & $0.167 \mathrm{gram} / \mathrm{cm}^{3}$ & $0.143 \mathrm{gram} / \mathrm{cm}^{3}$ \\
\hline Worn out mass & $0.09 \mathrm{gram} / \mathrm{min}$ & $0.11 \mathrm{gram} / \mathrm{min}$ \\
\hline
\end{tabular}

The cashew net shell husk composite is found to have better abrasion resistance than the silica sand composite.

6.6. Comparison between silica sand composite $\left(1^{\text {st }}\right.$ trial) and cashew nut shell husk composite

A comparison was made between the silica sand composite which is used for industrial flooring and cashew nut shell husk composite. The comparison was done in tensile, composite and flexural strengths.

Table -4

\begin{tabular}{|l|l|l|l|l|}
\hline Material & Tensile Strength & Compression Strength & Flexural Strength & Rate Of Abrasion \\
\hline Cashew nut shell & $13 \mathrm{MPa}$ & $61 \mathrm{MPa}$ after 2 & $22 \mathrm{MPa}$ & $0.09 \mathrm{gram} / \mathrm{min}$ \\
\hline husk & & days & & \\
\hline Silica sand composite & $15 \mathrm{MPa}$ & $70 \mathrm{MPa}$ after 5 & $32 \mathrm{MPa}$ & $0.11 \mathrm{gram} / \mathrm{min}$ \\
\hline & & days & & \\
\hline
\end{tabular}

The compression strength of Silica sand composite after 5 days is found to be $70 \mathrm{Mpa}$. The compression strength of cashew shell husk composite is expected to reach $70 \mathrm{Mpa}$ after five days where it was $61 \mathrm{Mpa}$ after two days. The verdict can be made from the comparison table that, the cashew nut shell husk composite has better abrasion resistance than the silica sand composite. It also holds good compressive strength, hardness required for industrial flooring.

\section{Conclusion}

From the above reports and evidences, we can conclude that the cashew nut shell husk composite is:

Antiskid in nature

Excellent load bearing

Low maintenance costs

Abrasion resistant and chemical resistant

This composite can be applied in areas like Chemical industries, Heavy industries, Workshops, Machine shops, Ramps and wherever high wear and tear resistance and chemical resistant floor is required. The abrasion resistance, surface friction and compression strength of our composite makes it significant.

\section{Future Scope}

The usage of Cashew Husk cannot be restricted to the application of flooring. As it a combination of base, hardener and filler, a variety of properties can be obtained by changing the fillers. Wide range of experiments can be done. One among them is the manufacture of brake pad using cashew husk. Using silica sand and cashew husk as filler gave good abrasion, compression and hardness with some flaws like porosity and reluctance to temperature exposure. So, we opine that using high density fillers, materials that can increase friction coefficient and also other means of binding can obtain the best unconventionally manufactured brake pad. It has been recently predicted that the most important technologies of the future that incorporate natural fiber composite materials will be natural fibers for injection moulded products $(32 \%)$ followed by natural fibers with a bio plastic matrix (19\%) and modified fibers for use in advanced applications (19\%). 


\section{References}

[1]. Li XH, Meng YZ, Wang SJ, Rajulu AV, Tjong SC. "Completely biodegradable composites of poly (propylene carbonate) and short, lignocellulose fabric hildegardia populifolia.” JPolym Sci: Part B; Volume 42, (2004): p. 666675.

[2]. Shibata M, Takachiyo K, Ozawa K, Yosomiya R, Takeishi H. "Biodegradable polyester composites reinforced with short abaca fiber.” J Appl Polym Sci; Volume 85, (2002): p. 129-138.

[3]. Iannace S, Nocilla G, Nicolais L. "Biocomposites based on sea algae fibers and biodegradable thermoplastic matrices.” J Appl Polym Sci; Volume 73, (1999): p. 583-92.

[4]. Shibata M, Ozawa K, Teramoto N, Yosomiya R, Takeishi H. "Biocomposites made from short abaca fiber and biodegradable polyester." Macromol Mater Eng; Volume 288, (2003): p. 35-43.

[5]. Luo S, Netravali A.N., "Interfacial and mechanical properties of environment-friendly,green" composites made from pineapple fibers and poly (hydyoxybutyrate-co-valerate) resin." J Mater Sci; Volume 34, (1999): p. 3709-3719.

[6]. Rout J, Misra M, Tripathy SS, Nayak SK, Mohanty AK. "The influence of fiber treatment on the performance of coir-polyester composites." Compos Sci Technol; Volume 61, (2001): p. 1303-1310.

[7]. Bisanda ETN. "The effect of alkali treatment on the adhesion characteristics of sisal fibres." Appl Comp Mater; Volume 7, (2000): p. 331-339.

[8]. Gassan J, Bledzki A.K. "Possibilities for improving the mechanical properties of jute/epoxy composites by alkali treatment of fibres." Compos Sci Technol.; Volume 59, (1999): p. 1303-1309.

[9]. Usmani M.Arthur, Salyer O. Ival, Ball L. George, Schwendeman L. James, "Bagasse-Fiber-Reinforced Composites." Journal of Elastomers and Plastics, Volume 13, No. 1, (1981): p. 46-73.

[10]. Monteiro S.N.; Rodriquez R.J.S.; De Souza M.V., D'Almeida J.R.M., "Sugar Cane Bagasse Waste as Reinforcement in Low Cost Composites", Advanced performance Material, Volume 5, No.3, (December 1998): p. 183-191. 73

[11]. Vazquez A., Dominguez V. A., Kenny J. M., "Bagasse Fiber-Polypropylene Based Composites." Journal of Thermoplastic Composite Materials.” Volume 12, No. 6, (1999): p.477-497.

[12]. Hassan M.L., Rowell R.M., Fadl N.A., Yacoub S.F. and Chrisainsen A.W. "Thermo plasticization of Bagasse. I. Preparation and Characterization of Esterified Bagasse Fibers." Journal of applied polymer science, Volume 76, (2000): p. 561-574.

[13]. Hassan M.L., Rowell R.M., Fadl N.A., Yacoub S.F. and Chrisainsen A.W. "Thermo plasticization of Bagasse. II. Dimensional Stability and Mechanical Properties of EsterifiedBagasse Composite." Journal of applied polymer science, Volume 76, (2000): p. 575-586. 